\title{
The Effect of Massive Binaries on Stellar Populations and Supernova Progenitors
}

\author{
John J. Eldridge ${ }^{1}$, Robert G. Izzard ${ }^{2}$ and Christopher A. Tout ${ }^{1}$ \\ ${ }^{1}$ University of Cambridge, Institute of Astronomy, The Observatories, Madingley Road, \\ Cambridge CB3 0HA, UK \\ e-mail (J.J. Eldridge): jje@ast.cam.ac.uk \\ ${ }^{2}$ Sterrekundig Instituut Utrecht, Postbus 80000, 3508 TA Utrecht, The Netherlands
}

\begin{abstract}
We have calculated a large set of detailed binary models and used them to test the observed stellar population ratios that compare the relative populations of blue supergiants, red supergiants and Wolf-Rayet stars at different metallicities. We have also used our models to estimate the relative rate of type Ib/c to type II supernovae. We find, with an interacting binary fraction of about two thirds, that we obtain better agreement between our models and observations than with single stars. We discuss the use of models in determining the nature of supernova progenitors and show the surprising result that many type Ib/c supernova progenitors are less luminous and less massive in our models than the observed population of Wolf-Rayet stars.
\end{abstract}

Keywords. binaries: close - stars: evolution - supernovae: general - stars: Wolf-Rayet

\section{Introduction}

When we try to match the observed properties of massive stars with single star models we have always found a poor fit. For example, the ratio of the number of blue supergiants to red supergiants and its variation with metallicity cannot be reproduced (Langer \& Maeder 1995; Massey \& Olsen 2003). The sources of this disparity could be due to our limited models of convection or the lack of rotation in our stellar models (Maeder \& Meynet 2001). Another possibility is that a large number of these stars have binary companions. It is well known that this can change stellar populations substantially (Vanbeveren, Van Rensbergen \& De Loore 1998).

We have produced a large set of binary stellar models to predict their effect on the relative populations of blue supergiants (BSGs), red supergiants (RSGs) and Wolf-Rayet (WR) stars. The advantage of our study (Eldridge, Izzard \& Tout 2008) is that we model the binary interactions in a detailed stellar evolution code rather than approximating the evolution as in rapid population synthesis that use tables or equations fitted to detailed models (Hurley, Tout \& Pols 2002). These detailed models ensure that more uncertain phases of evolution, such as when the hydrogen envelope is close to being removed, are treated as accurately as possible. This is vital when we attempt to determine a stellar type for a model and its respective lifetime.

In this proceedings we first summarize our results as described in Eldridge, Izzard \& Tout (2008), where we compare the relative stellar populations predicted by our code with observed populations. We then discuss how our results indicate that a large number of type Ib/c supernovae, those devoid of hydrogen, may not have WR stars as their progenitors. 


\section{Stellar population ratios}

In Figures 1, 2 and 3 we compare observations of three stellar population ratios at various metallicities to ratios predicted from our models. The observed ratios are calculated by observing galaxies and counting the number of each type of star and dividing the number in one population by another (Massey \& Olsen 2003). The model predictions are calculated by first determining how long each model spends as either a BSG, RSG or WR star. We use the definitions of BSGs and RSGs from Massey \& Olsen (2003) and the definitions for WR stars from Maeder \& Meynet (1994). In addition we also require that a WR star must have $\log _{10}\left(L / \mathrm{L}_{\odot}\right) \geqslant 4.9$ to be consistent with the luminosity limit for BSGs and RSGs.

With the BSG, RSG and WR lifetimes we then use an initial mass function and assume a constant star formation rate to calculate the relative populations. For the binary population we take flat distributions in the ratio of the secondary to the primary mass and the logarithm of the initial separation. We find that about two thirds of our binaries interact while the remaining third evolve as single stars.

Figure 1 shows the relative number of BSGs, which are main sequence stars, to RSGs, post-main sequence stars that still have their hydrogen envelopes. Even though there are only two points on the figure, it demonstrates that binary models better match these observations. This is for two reasons. First more RSGs lose their hydrogen envelopes than for single stars by interaction with their companion and thus the number of RSGs decreases. Secondly, more massive stars are formed from low-mass companions accreting matter or by stellar mergers. Therefore the BSG population is increased.

The RSGs that lose their hydrogen envelopes become helium stars or WR stars. Figure 2 shows how the number of $\mathrm{O}$ stars (the hottest BSGs) against the number of WR stars, varies with metallicity. We see that the increased WR population does improve agreement with observations. Also the binary line agrees with predictions of the Geneva

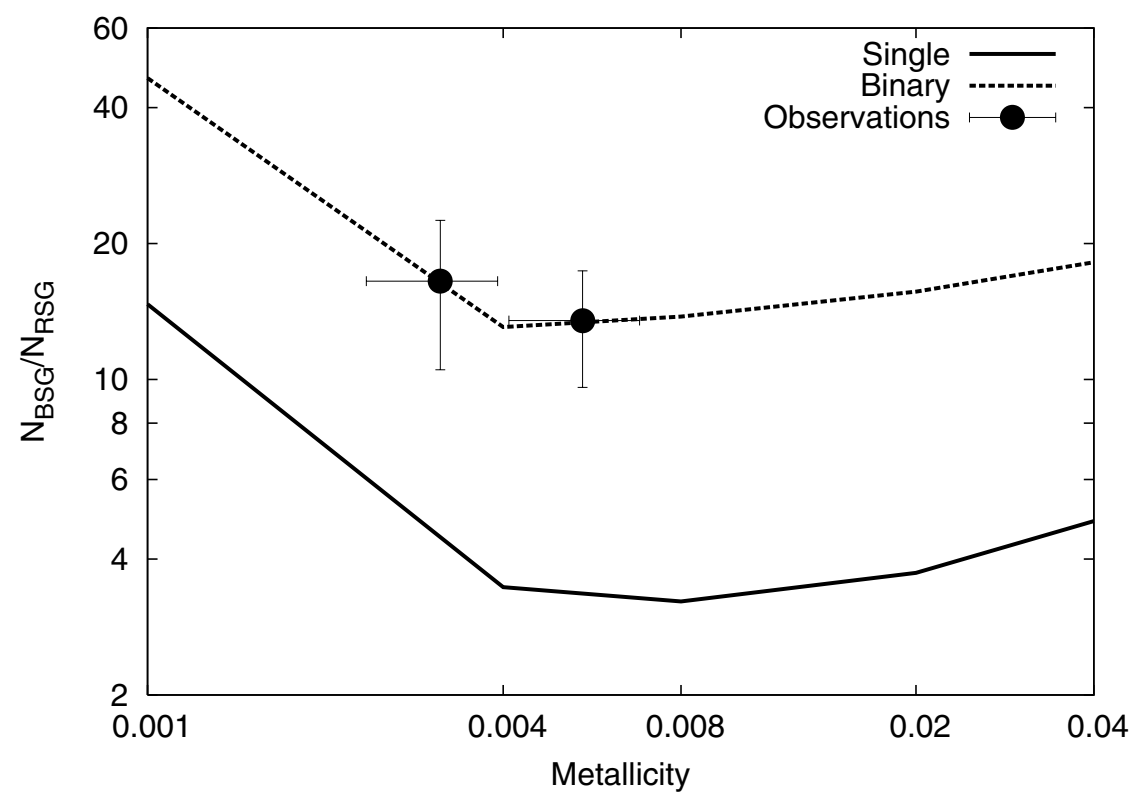

Figure 1. Ratio of the numbers of blue supergiants to red supergiants versus metallicity. Observations are taken from Massey \& Olsen (2003). The solid line is from our single star models while the dashed line is from our binary models. 
rotating models Meynet \& Maeder (2005) so rotation and binaries appear to have the same effect on this stellar population ratio.

Binaries do not improve agreement for all the ratios. In Figure 3 we show the relative number of RSGs to WR stars. We see that neither the single nor binary star model predictions match the observed trend. This it at odds with the match in the previous two figures which show good agreement. Including missing details from our models (such as rotation) may improve agreement. However it is more likely that because the ratio is based on a small number of observed RSGs, especially when metallicity is greater than $Z=0.008$ our assumptions in calculating the predicted ratios are not appropriate. For example with only a few stars we cannot be certain we are sampling the IMF fully nor looking at a sample with constant star formation.

In summary binary models improve agreement between predicted and observed stellar population ratios. The agreement is not always perfect and extra details still need to be added to our models.

\section{Relative supernova rates}

The final outcomes of massive stellar evolution, supernovae ( $\mathrm{SNe}$ ), can also be used to provide a constraint on stellar models because the SN type depends on the final stellar type. These events come in three broad types. Type Ia SNe are thought to be thermonuclear explosions of carbon-oxygen white dwarfs and are not of relevance here. The remaining two types are core-collapse SNe where either an oxygen-neon or iron core is formed which collapses when electron degeneracy pressure or nuclear burning can no longer provide support against gravitational collapse. This collapse releases a tremendous

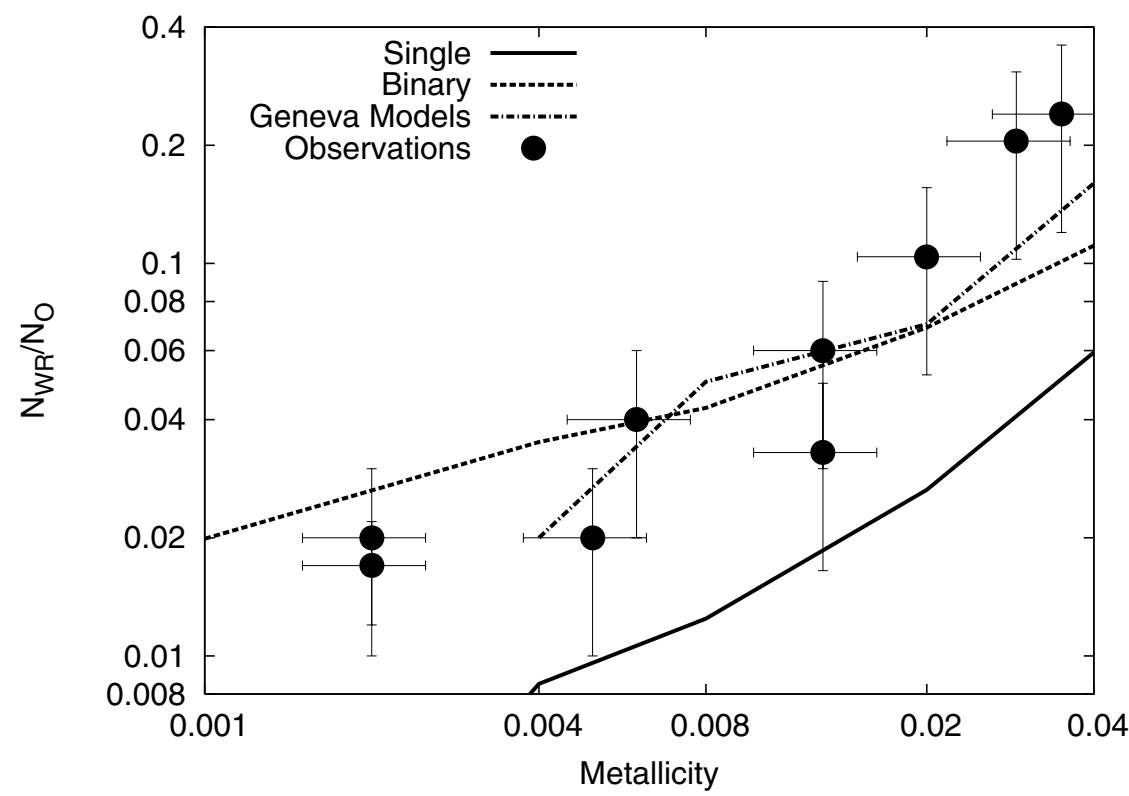

Figure 2. Ratio of the numbers of Wolf-Rayet stars to O-supergiants versus metallicity. Observations are taken from Maeder \& Meynet (1994). The solid line is our single star models while the dashed line is our binary models. The dashed-dotted line is from the Geneva models (Meynet \& Maeder 2005). The y-axis error bars are an assumed error of 50 percent of the values given by Maeder \& Meynet (1994). 
amount of energy that is transferred into the envelope causing the subsequent SN (Heger et al. 2003; Eldridge \& Tout 2004).

While there are many subtypes of SN the broadest definitions are type II, when hydrogen is detected in the SN spectrum and type $\mathrm{Ib} / \mathrm{c}$ where hydrogen is undetected. The relative rate of type $\mathrm{Ib} / \mathrm{c}$ to type II SNe indicates the number of stars that experienced mass loss strong enough to remove their hydrogen envelope before core-collapse. Prantzos \& Boissier (2003) first showed that this ratio decreases with metallicity. However, they only estimated the SN metallicities from the host galaxy magnitude. Recently Prieto, Stanek \& Beacom (2008) presented a more detailed analysis, estimating the metallicity by spectroscopy. We compare both observed trends in Figure 4. They agree within the errors but Prieto, Stanek \& Beacom (2008) tend to have slightly lower ratios around solar metallicity and predict a much shallower evolution with metallicity. The lowest metallicity bin is uncertain and is based on one type $\mathrm{Ib} / \mathrm{c} \mathrm{SN}$.

Comparing to the model predictions we see that the binary models and Geneva rotating models provide the best agreement. A large fraction of the type Ib/c progenitors are not WR stars but are helium stars with $M<5 \mathrm{M}_{\odot}$. In the stellar population ratios above for a star to be a WR star we required that $\log \left(L / \mathrm{L}_{\odot}\right) \geqslant 4.9$ which is similar to the least luminous WR star that has been observed. However from our binary models there are many stars that lose their hydrogen envelopes and explode as type Ib/c SN but are not WR stars, this has been discussed by others (Vanbeveren, Van Rensbergen \& De Loore 1998; Pols \& Dewi 2002). The question then becomes where are these helium stars or low-mass WR stars. They remain unobserved. They would be similar to stars such as those described by Wood \& Lockley (2000) and Oliveira, Steiner \& Cieslinski (2003).

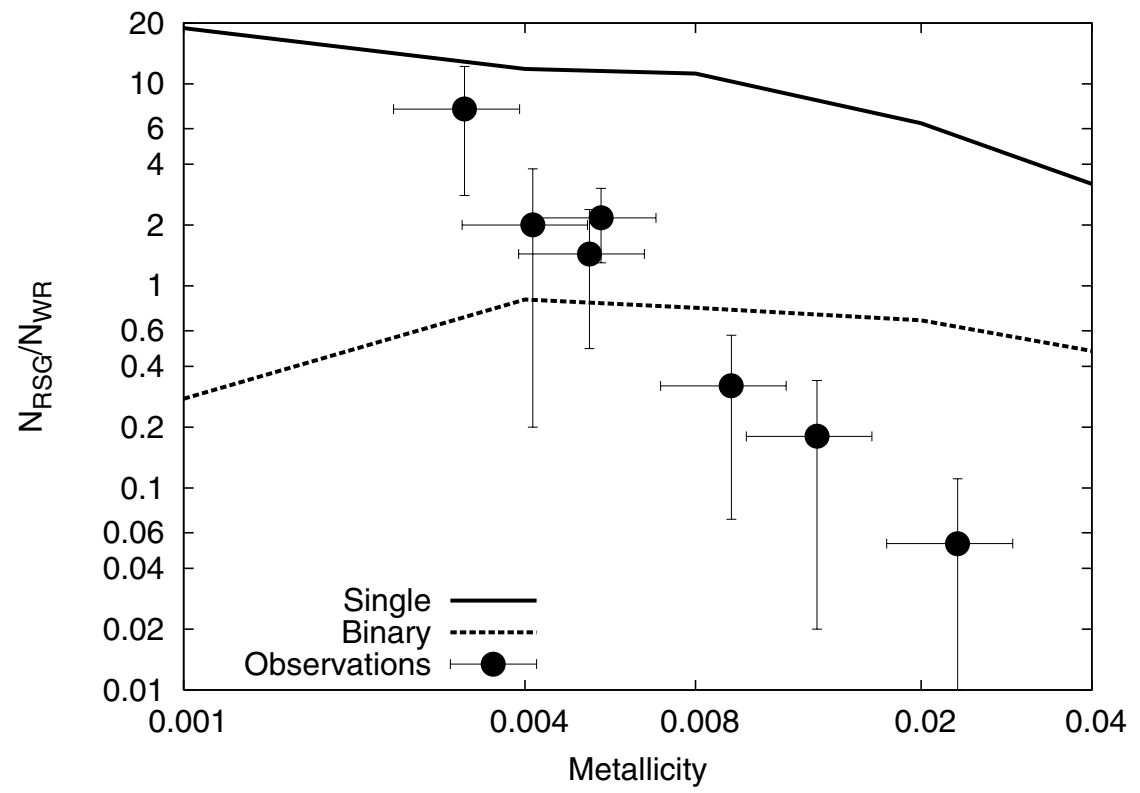

Figure 3. Ratio of the number of red supergiants to Wolf-Rayet stars versus metallicity. The observations are taken from Massey (2003). The solid line is from our single star models while the dashed line is from our binary models. 


\section{Progenitors of type $\mathrm{Ib} / \mathrm{c}$ supernovae}

To support the above conclusion that many type Ib/c SN progenitors must be lowmass helium stars we have compared our binary models to the detection limits placed on the progenitors by studies such as that of Crockett et al. (2007). If we compare the deepest B-band magnitude limit for a type Ib/c SN to date, that of SN 2002ap, to model B-band magnitudes calculated by combining our models and the WR atmosphere models of Hamann, Gräfener \& Liermann (2006), then we find that helium/WR stars more massive than $3 \mathrm{M}_{\odot}$ would have been observed while only the less massive stars would remain undetected. This confirms the conclusion of Crockett et al. (2007) that if the progenitor was a normal massive WR star it would have been observed in the preexplosion images. The number of such non-detections is growing and therefore a large number of type Ib/c SN may not have WR progenitors as previously thought.

\section{For the future}

The next step with this large set of binary models is to find further problems to apply it to. One extension is to model an instantaneous burst of star formation rather than continuous star formation.

Where are these helium stars or low-mass WR stars? We infer they exist but answering this question requires an observational solution. The reason why they have remained unobserved to date is that they may be in binaries and may be hidden by their companions, or they could be copious producers of dust and therefore obscured. Regions where they may exist must be identified and observed more extensively.

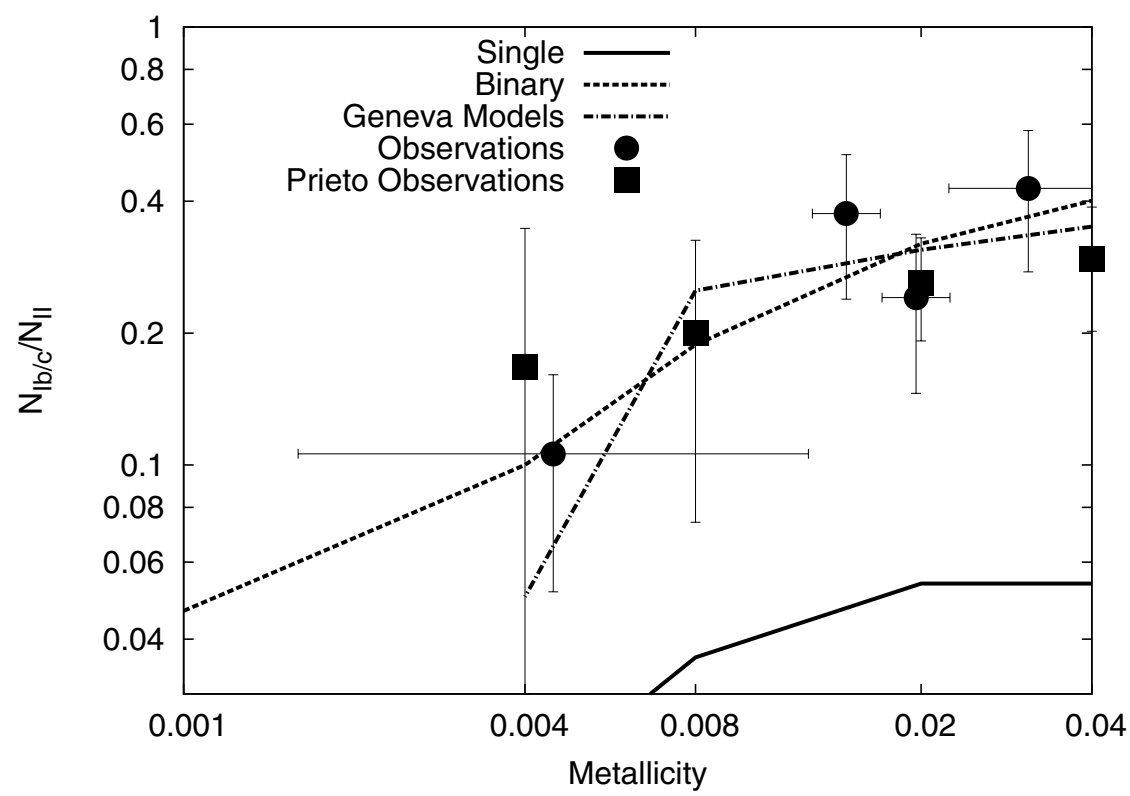

Figure 4. The observed and predicted ratios of the type $\mathrm{Ib} / \mathrm{c}$ supernova rate to the type II supernova rate. Observations are taken from Prantzos \& Boissier (2003) (circles) and Prieto, Stanek \& Beacom (2008) (squares). The Geneva model predictions are taken from Meynet \& Maeder (2005). The line is for their rotating models. 


\section{Acknowledgements}

JJE carried out as part of the "Understanding the lives of massive stars from birth to supernovae" EURYI Award. CAT thanks Churchill College, Cambridge for his Fellowship. RGI thanks the NWO for his current fellowship in Utrecht.

\section{References}

Crockett R. M., Smartt S. J., Eldridge J. J., et al. 2007, MNRAS, 381, 835

Eldridge J. J. \& Tout C. A. 2004, MNRAS, 353, 87

Eldridge J. J, Izzard R. G., \& Tout C. A. 2008, MNRAS, 384, 1109

Hamann W.-R., Gräfener G., \& Liermann A. 2006, A\&SA, 457, 1015

Heger A., Fryer C. L., Woosley S. E., et al., 2003 ApJ, 591, 288

Hurley J. R., Tout C. A., \& Pols O. R. 2002, MNRAS, 329, 897

Langer N. \& Maeder A. 1995, A\&A, 295, 685

Maeder A. \& Meynet G. 1994, A\&A, 287, 803

Maeder A. \& Meynet G. 2001, A\&A, 373, 555

Massey P. 2003, ARA\&A, 41, 15

Massey P. \& Olsen K. A. G. 2003, $A J, 126,2867$

Meynet G. \& Maeder A. 2005, A\&A, 429, 581

Oliveira A. S., Steiner J. E., \& Cieslinski D. 2003, MNRAS, 346, 963

Pols O. R. \& Dewi J. D. M. 2002, PASA, 19, 233P

Prantzos N. \& Boissier S. 2003, A\&A, 406, 259

Prieto J. L., Stanek K. Z., \& Beacom J. F. 2008, ApJ, 673, 999

Vanbeveren D., Van Rensbergen W., \& De Loore C. D. 1998, The Brightest Binaries (Dordrecht: Springer) Astrophys. Space Science Lib., v. 232,

Wood J. H. \& Lockley J. J. 2000, MNRAS, 313, 789

\section{Discussion}

MAEDER: It would be interesting to combine the results of single and binary evolution with ratios between them based on the binary rates given yesterday by Tony Moffat. Now, as a side remark, I am surprised by the smallness of the blue loops of your tracks in the HR diagram.

ELDRIDGE: The ratio of the single and binary populations is fixed by the assumed initial binary parameter distribution. The number of interacting binaries we find is similar to the numbers given by Tony Moffat yesterday. We could consider this an independent check of to determine single/binary ratio. To answer the blue loops remark I should say myself and Richard Stancliffe are very interested in them and are looking into them. We are looking into them observationally and theoretically.

GAYLEY: The nomenclature of "single star" and "binary" is confusing because theorists tend to mean objects whose evolution is altered, and observers tend to mean objects that offer unique observational diagnostics. So I'd like to enter a plea that we routinely distinguish three types, rather than two: evolutionary binaries, observational binaries and stars of unknown or unobservable binarity. Note these overlap: interacting binaries are the overlap of the first two, runaways are the overlap of the first and the third.

ELDRIDGE: I think this is a great idea. One thing I intend to do is to calculate such details as how many binaries we would expect to observe, how many stars we might not observe as binaries and also details such as runaways. But the situation is confusing and you are right that we must be clear about how we apply the adjective "binary" to stars. 